\title{
POTENTIAL MEASURES OF ONE-SIDED MARKOV ADDITIVE PROCESSES WITH REFLECTING AND TERMINATING BARRIERS
}

\author{
JEVGENIJS IVANOVS, * University of Lausanne
}

\begin{abstract}
Consider a one-sided Markov additive process with an upper and a lower barrier, where each can be either reflecting or terminating. For both defective and nondefective processes, and all possible scenarios, we identify the corresponding potential measures, which help to generalize a number of results for one-sided Lévy processes. The resulting rather neat formulae have various applications in risk and queueing theories, and, in particular, they lead to quasistationary distributions of the corresponding processes.
\end{abstract}

Keywords: Markov modulation; exit from an interval; resolvent; time reversal; transient analysis; quasistationary; quasistationary distribution

2010 Mathematics Subject Classification: Primary 60G51

Secondary $60 \mathrm{~J} 25$

\section{Introduction}

A Markov additive process (MAP) in continuous time is a natural generalization of a Lévy process. They have various applications in queueing, risk theory, and financial mathematics; see, for example, [2]. It can be seen as a Lévy process in a Markov environment, which provides rich modeling possibilities.

The main exit problems for spectrally negative MAPs were solved in [17], including onesided and two-sided exits, as well as exits for reflected processes. Following these developments we consider a spectrally negative MAP with two barriers (upper and lower), where each barrier can be either terminating or reflecting, and identify the corresponding potential measures (also known as resolvent measures). It is assumed that a process is stopped (killed) when it passes over a terminating barrier, i.e. above an upper or below a lower terminating barrier. Barriers at $\infty$ and $-\infty$ are also allowed and these correspond to a model with less than two barriers.

Potential measures have numerous applications which can be anticipated from the general theory of Markov processes. In the case of spectrally negative Lévy processes various potential measures can be given in an explicit form; see [21, Section 8.4] and the references therein. It should also be noted that potential measures and their densities readily lead to the distribution of the corresponding process at an exponential time; we will be discussing more about the MAP setting in what follows. Recent progress in the theory of spectrally negative MAPs allows us to present rather neat equations and short proofs.

Certain potential measures of MAPs are known in the literature. Almost all the known results concern Markov modulated Brownian motion (MMBM) or a MAP with phase-type jumps, which can be represented as an MMBM with an expanded environmental process. It should be noted that an MMBM allows for a much simpler analysis because of the absence of jumps.

Received 3 October 2013; revision received 16 January 2014.

* Postal address: Department of Actuarial Science, Faculty of Business and Economics, University of Lausanne, Quartier UNIL-Dorigny, 1015 Lausanne, Switzerland. Email address: jevgenijs.ivanovs@unil.ch 
The transforms of occupation times up to an exit point from an interval were obtained in [9]. Potential measures of the free process and one-sided reflection process were obtained in [10]. Moreover, some results for the two-sided reflection process appeared in [15]. The potential measure of a spectrally negative MAP (under certain assumptions) in the case of a single lower terminating barrier was obtained in [14], using a very different approach based on algebraic operations of matrix operators. Finally, some very interesting applications of potential measures to insurance risk can be found in [10], [14], and [26].

In Section 2 we define a MAP and set up the associated notation. In Section 3 we formulate the problem and discuss the relation between potential measures and quasistationary distributions. In Section 4 we review some basic results from the exit theory of MAPs and define related matrices and matrix-valued functions, which will be necessary to formulate our main results. In Section 4 we review occupation densities, which constitute the main tool for deriving potential densities in the case of terminating barriers, which are dealt with in Section 5. Time reversal, discussed in Section 6, is the main tool used in Section 7 in deriving potential densities for reflected processes. Our results are extended to nondefective processes in Section 8, where we also obtain certain stationary distributions as a side result. Concluding remarks are given in Section 9.

The results on potential measures (and, hence, on quasistationary distributions) are spread among Theorem 1, Theorem 2, Corollary 2, and Corollary 3. In addition, in Corollary 4 we present stationary distributions. Finally, this paper contains some useful identities concerning time-reversed processes and various limits, contained in Proposition 1, Corollary 1, and Lemma 1.

\section{Preliminaries}

A MAP is a bivariate Markov process $(X, J)=\{(X(t), J(t)): t \geq 0\}$, where $X$ is an additive level component and $J$ represents the environment; see [2, Section XI.2a]. It is assumed that $(X, J)$ is adapted to some right-continuous, complete filtration $\left\{\mathcal{F}_{t}: t \geq 0\right\}$, and that $J$ is an irreducible Markov chain on a finite number of states, say $n$. The defining property of a MAP is that, for any $t \geq 0$ and any $i=1, \ldots, n$ conditionally on $\{J(t)=i\}$, the process

$$
\{(X(t+s)-X(t), J(t+s)): s \geq 0\}
$$

is independent of $\mathcal{F}_{t}$ and has the law of $\{(X(s)-X(0), J(s)): s \geq 0\}$ given $\{J(0)=i\}$. In other words, the environment governs the increments of the level process. Importantly, this property also holds when $t$ is replaced by any stopping time $\tau$; note that $\{J(\tau)=i\}$ implicitly assumes that $\tau<\infty$. We allow for defective (or killed) processes, i.e. we add an additional absorbing cemetery state to the environment without writing it explicitly. Hence $\{J(t)=i\}$ also means that the process has survived up to time $t$. In the special case when $n=1$ we obtain a Lévy process which may be killed at an independent exponential time.

Using the Markov additive property requires repeated conditioning on the state of the environment, which leads to matrix algebra and justifies the following notation. For a random variable $Y$, we write $\mathbb{E}_{x}[Y ; J(t)]$ to denote an $n \times n$ matrix with $i j$ th element

$$
\mathbb{E}_{x, i}(Y ; J(t)=j)=\mathbb{E}\left(Y 1_{\{J(t)=j\}} \mid X(0)=x, J(0)=i\right) .
$$

Similarly, for an event $A$, we write $\mathbb{P}_{x}[A, J(t)]=\mathbb{E}_{x}\left[1_{A} ; J(t)\right]$ for the corresponding matrix of probabilities. Moreover, subscript $x$ can be omitted when $x=0$. Finally, the identity and the zero matrices are denoted by $\mathbb{I}$ and $\mathbb{O}$, respectively, and $\Delta_{v}$ stands for a diagonal matrix with a vector $v$ on the diagonal. 
There exists a matrix-valued function $F(\alpha)$, which characterizes the law of the process $(X, J)$, and, in particular,

$$
\mathbb{E}\left[\mathrm{e}^{\alpha X(t)} ; J(t)\right]=\mathrm{e}^{F(\alpha) t}, \quad \alpha, t \geq 0 .
$$

Note that $F(0)$ is the transition rate matrix of $J$, and, hence, our MAP is nondefective if and only if $F(0) \mathbf{1}=\mathbf{0}$, where $\mathbf{1}$ and $\mathbf{0}$ denote column vectors of $1 \mathrm{~s}$ and $0 \mathrm{~s}$, respectively. In this case let a row vector $\pi$ be the stationary distribution of $J$, and let $\mu=\mathbb{E}_{\pi} X(1)$ be the asymptotic drift. According to whether $\mu<0, \mu>0$, or $\mu=0$ the process $X(t)$ (as $t \rightarrow \infty)$ tends to $-\infty, \infty$, or oscillates between $-\infty$ and $\infty$, respectively; see [2, Proposition XI.2.10].

\section{Potential measures}

Assume that $X(0)=0$, and that barriers are placed at $-a$ and at $b$, where $a, b \in[0, \infty]$ (not simultaneously 0); otherwise, we can simply shift the picture. We write $|-a, b|$, $\mid-a, b],[-a, b \mid$, and $[-a, b]$ to denote different scenarios, where 'I' denotes termination and '[' or ']' denotes reflection at the corresponding barriers. In particular, $X_{[-a, b \mid}(t)$ is a process reflected at $-a$ and terminated at $b$, i.e. upon exiting the interval $(-\infty, b]$. In the case when a barrier is placed at $-\infty$ or $\infty$, i.e. there is no barrier, we write $(-\infty, \infty),(-\infty, b \mid,(-\infty, b$, $\mid-a, \infty,[-a, \infty)$ to denote all possible scenarios, where the first corresponds to a free process, the second denotes termination upon exiting the interval $(-\infty, b]$, and so forth. For a rigorous definition of reflection, both one-sided and two-sided; see, for example, [2] and [19]. Here we only recall the simplest cases $X_{[0, \infty)}(t)=X(t)-\underline{X}(t)$ and $X_{(-\infty, 0]}(t)=X(t)-\bar{X}(t)$, where $\underline{X}(t)$ and $\bar{X}(t)$ are the running infimum and supremum, respectively, and note that any reflecting barrier locally acts in a similar way; cf. [2, Chapter XIV.3].

For each of the above scenarios $I$ (e.g. (1) $I=\mid-a, b]$ ) we consider the corresponding potential measure (a matrix of measures)

$$
U_{I}(A)=\int_{0}^{\infty} \mathbb{P}\left[X_{I}(t) \in A, J(t)\right] \mathrm{d} t=\left(\mathbb{E}_{i} \int_{0}^{\infty} 1_{\left\{X_{I}(t) \in A, J(t)=j\right\}} \mathrm{d} t\right),
$$

where $A \in \mathscr{B}[-a, b]$. It turns out that in all the cases the measure $U_{I}(A)$ has a density $u_{I}(x)$ on $(a, b)$ with respect to the Lebesgue measure. We identify this density and compute the point masses at $-a$ and $b$. We will show that there is never a point mass at the level of a terminating barrier, and, hence, we only specify the point masses for the levels of reflecting barriers. One of the possible uses of potential measures is given by the occupation density equation

$$
\mathbb{E}_{i} \int_{0}^{\infty} f(X(t), J(t)) \mathrm{d} t=\sum_{j} \int_{\mathbb{R}} f(x, j) U_{i j}(\mathrm{~d} x),
$$

where $f \geq 0$ is a measurable function, which is equal to 0 for the cemetery state of the environment. Various interesting applications of this equation applied to insurance risk are presented in [14].

Recall that we allow for defective MAPs, in which case we can think that time elapses only up to the killing epoch (when $J$ enters the cemetery state). A defective MAP can be seen as a nondefective MAP killed at some rate $q_{i} \geq 0$ while $J$ is in $i$ for all $i=1, \ldots, n$. A special case arises when $q_{i}=q$ for all $i$, i.e. a MAP is killed at an independent exponential time $e_{q}$ of rate $q$. Then

$$
\mathbb{P}\left[X_{I}\left(e_{q}\right) \in A, J\left(e_{q}\right)\right]=q \int_{0}^{\infty} \mathrm{e}^{-q t} \mathbb{P}\left[X_{I}(t) \in A, J(t)\right] \mathrm{d} t=q U_{I}^{q}(A),
$$


where superscript $q$ is used to distinguish between objects corresponding to a MAP and its killed version. This relation can be generalized in the following way. Let $\boldsymbol{q}=\left(q_{1}, \ldots, q_{n}\right)$ be a vector of killing rates, and let $T$ be the killing time, i.e. the lifetime of the Markov chain $J$, implying that $T$ has a corresponding phase-type distribution. A standard argument shows that

$$
\mathbb{P}\left[X_{I}(T) \in A, J(T)\right]=\int_{0}^{\infty} \mathbb{P}^{\boldsymbol{q}}\left[X_{I}(t) \in A, J(t)\right] \mathrm{d} t \Delta_{\boldsymbol{q}}=U_{I}^{\boldsymbol{q}}(A) \Delta_{\boldsymbol{q}} .
$$

We can think about an independent Poissonian observer whose rate, $q_{i}$, depends on the environment. Then $U_{I}^{q}(A) \Delta_{q}$ gives the distribution of the process at his first observation epoch $T$. This idea appears in [10], where, in addition, it is shown how to treat the case where $T$ is an arbitrary phase-type variable independent of $J$.

\section{Exit theory review}

Throughout this paper, it is assumed that the level component $X$ has no positive jumps, and that, for each $i$, the process $X$ given $\{J(0)=i\}$ visits $(0, \infty)$ before the switch to the environment with positive probability (none of the underlying Lévy processes is a downward subordinator). The second assumption, also appearing in [17], allows us to simplify our notation greatly and to avoid unpleasant technical difficulties. Let us briefly review the exit theory of such MAPs.

For $x \geq 0$, define the first passage times

$$
\tau_{x}^{+}=\inf \{t \geq 0: X(t)>x\}, \quad \tau_{-x}^{-}=\inf \{t \geq 0: X(t)<-x\} .
$$

In addition, we define the first hitting time of a level $x \in \mathbb{R}$ by

$$
\tau_{\{x\}}=\inf \{t>0: X(t)=x\} .
$$

It is known that $\tau_{x}^{+} \leq \tau_{\{x\}}$ and $\tau_{-x}^{-} \leq \tau_{\{-x\}}$ for $x \geq 0$ almost surely (a.s.), i.e. $X$ cannot hit a level without immediately passing over it, which can be seen from the small-time behavior of Lévy processes; see also [17, Proposition 7].

\subsection{Occupation densities}

Let us consider $L(x, j, t)$, the occupation density of $(X, J)$ at $(x, j)$ up to time $t$, which is defined in [17] based on the corresponding theory for Lévy processes. Firstly, $L(x, j, t)$ always exists under our assumptions. It is nonnegative, measurable in $x$, and, for all $t \geq 0$ and all measurable $f \geq 0$, satisfies

$$
\int_{0}^{t} f(X(s), J(s)) \mathrm{d} s=\sum_{j} \int_{\mathbb{R}} f(x, j) L(x, j, t) \mathrm{d} x \quad \text { a.s. }
$$

where $f$ is 0 for the cemetery state. Compare this occupation density formula to (2). Note that $L$ leads to a more refined formula, but that is valid only for a free process and moreover $L$ lacks explicit expression.

Remark 1. Let us briefly present the construction of the occupation density for MAPs; see [5], [17] for details. Although it is not needed in this paper, it may help in the general understanding. 
We have

$$
\begin{aligned}
& L(x, j, t)=\lim _{\varepsilon \downarrow 0} \frac{1}{2 \varepsilon} \int_{0}^{t} 1_{\{|X(s)-x|<\varepsilon, J(s)=j\}} \mathrm{d} s, \quad 0 \text { is regular under } \mathbb{P}_{j}, \\
& L(x, j, t)=\frac{1}{d_{j}} \#\{s \in[0, t): X(s)=x, J(s)=j\}, \quad 0 \text { is irregular under } \mathbb{P}_{j},
\end{aligned}
$$

where in the second case $d_{j}>0$ is the deterministic drift coefficient of $X$ when $J$ is in state $j$.

Importantly, for every $x \in \mathbb{R}$ and every $j,\{L(x, j, t): t \geq 0\}$ is a $\mathcal{F}_{t}$ adapted process, which increases only when $X=x$, and $J=j$, and inherits the following additive property. Consider a stopping time $\tau$ such that $X(\tau)=y \in \mathbb{R}$ on $\{J(\tau)=i\}$ for some $y$ and $i$. Then on the event $\{J(\tau)=i\}$, the shifted process $\{L(x, j, \tau+s)-L(x, j, \tau): s \geq 0\}$ is independent of $\mathcal{F}_{\tau}$ and has the law of $\{L(x-y, j, s): s \geq 0\}$ given $\{J(0)=i\}$.

\subsection{The fundamental matrices}

Define a matrix $H$ with elements $H_{i j}=\mathbb{E}_{i} L(0, j, \infty)$, i.e. it is the matrix of expected occupation times at 0 . It is known that $H$ has finite positive entries and is invertible unless the process is nondefective and $\mu=0$; see [17, Remark 4.1]. A numerical procedure to compute $H$ was outlined in [1, Proposition 4.1]. In the case of an MMBM $H$ has an explicit form; see [10] and [16, Chapter 7.7].

Observe that $\left\{J\left(\tau_{x}^{+}\right), x \geq 0\right\}$ is a Markov chain with some transition rate matrix $G$, so that

$$
\mathbb{P}\left[J\left(\tau_{x}^{+}\right)\right]=\mathrm{e}^{G x}, \quad x \geq 0 .
$$

The matrix $G$ can be identified for a given $F(\alpha)$ using iterative or spectral methods; see [8], [11] and the references therein. We use the symbol $G$ to be consistent with the theory of discrete time skip-free upwards MAPs; see [2, Section XI.3]. In fact, there are numerous similarities between discrete- and continuous-time theories, which were explored in [18].

Next, for a defective process, we define

$$
R=H^{-1} G H .
$$

This definition can be extended to a nondefective case by letting all the killing rates converge to 0 , where the existence of this limit follows from Proposition 1. It can be shown that the matrices $R$ and $G$ have the same eigenvalues, all of which have a negative real part, unless the process is nondefective and $\mu \geq 0$ in which case there is a simple eigenvalue at 0 and the rest have negative real parts.

Remark 2. Analogously to the discrete time case, the matrix $R$ plays a major role in various identities and can be seen as a counterpart of $G$. It is known that $G$ is the right solution of a certain matrix integral equation, whereas $R$ is the left solution of this equation; see [12], [18], where the latter also contains a probablistic interpretation of $R$. Recently, this matrix, under some assumptions on the process, has also appeared in [14].

Remark 3. Let us finally remark about simplifications when we consider a Lévy process, i.e. $J$ lives on a single state. Firstly, all the matrices become scalars, and, in particular, $F(0)=-q$, where $q \geq 0$ is the killing rate. Moreover, $G=R=-\Phi$, where $\Phi \geq 0$ is called the right inverse of the Laplace exponent $F(\alpha)$, i.e. it solves $F(\Phi)=0$. More precisely, $\Phi=0$ when $q=0$ and $\mu \geq 0$; otherwise $\Phi$ is a unique positive zero of $F(\alpha)$. Finally, according to [1, Proposition 4.1] we have $H=1 / F^{\prime}(\Phi)$, which indeed is finite unless $q=0$ and $\mu=0$. 


\subsection{The scale function}

Another important object in the exit theory for MAPs is a matrix-valued function $W(x)$ (often called the scale function), which is continuous on $[0, \infty)$ and is identified by the transform

$$
\int_{0}^{\infty} \mathrm{e}^{-\alpha x} W(x) \mathrm{d} x=F(\alpha)^{-1}
$$

for large enough $\alpha$; we put $W(x)=\mathbb{O}$ for $x<0$. It holds that $W(x)$ is nonsingular for $x>0$ and that

$$
\mathbb{P}\left[\tau_{b}^{+}<\tau_{-a}^{-} ; J\left(\tau_{b}^{+}\right)\right]=\mathbb{P}\left[\tau_{b}^{+}<\tau_{\{-a\}} ; J\left(\tau_{b}^{+}\right)\right]=W(a) W(a+b)^{-1} .
$$

The first equality arises because $X$ cannot hit a level $-a$ without immediately going below it, and if $X$ jumps below $-a$ then it has to hit it on the way to $b$.

Let us discuss differentiability of $W(x)$ for $x>0$. In [17, Theorem 5] it was shown that both left and right derivatives, $W_{-}^{\prime}(x)$ and $W_{+}^{\prime}(x)$, respectively, exist for all $x>0$, but may not coincide at countably many points. Furthermore, this result can be used to show that $\lim _{y \uparrow x} W_{+}^{\prime}(y)=W_{-}^{\prime}(x)$, and that $W_{+}^{\prime}(x)$ is Riemann integrable on any interval $[0, a]$. When $W_{+}^{\prime}(x)$ enters into density representation, we simply write $W^{\prime}(x)$.

In addition, we define a second scale function

$$
Z(x)=\mathbb{I}-\int_{0}^{x} W(y) \mathrm{d} y F(0),
$$

which is used in various exit identities. In general, the function $Z$ is defined as a function of two parameters $\alpha$ and $x$, but, for the purpose of this paper, the case in which $\alpha=0$ is sufficient.

\subsection{Some important identities}

The scale function is closely related to $H(x)$ defined by

$$
H_{i j}(x)=\mathbb{E}_{i} L\left(0, j, \tau_{x}^{+}\right) \text {for } x>0
$$

and $H(0)=\lim _{x \downarrow 0} H(x)$. In other words, $H(x)$ is a matrix of expected occupation densities at 0 for the time interval $\left[0, \tau_{x}^{+}\right)$. It was shown in [17, Equation 2] that

$$
W(x)=\mathrm{e}^{-G x} H(x)
$$

for $x \geq 0$. Note also that $H=H(\infty)$.

Remark 4. Note that $\tau_{x}^{+} \downarrow 0$ as $x \downarrow 0$ a.s., which implies that $W(0)=H(0)$ is a diagonal matrix. Its $(j, j)$ th element is either 0 or $1 / d_{j}$ according to the dichotomy in (5).

In the rest of this section we suppose that the process is defective. Then $H$ has finite entries and by the additive property of $L$ we have, for $x \geq 0$,

$$
H(x)=H-\mathbb{P}\left[J\left(\tau_{x}^{+}\right)\right] \mathbb{P}\left[J\left(\tau_{\{-x\}}\right)\right] H .
$$

So combining (8) and (9) we obtain

$$
\mathbb{P}\left[J\left(\tau_{\{x\}}\right)\right]=\mathrm{e}^{G x}-W(-x) H^{-1}
$$

for $x \leq 0$, but then it is clearly also true for $x>0$.

Finally, the following exit identity is known (see [17, Corollary 4]):

$$
\mathbb{P}\left[J\left(\tau_{-a}^{-}\right)\right]=Z(a)-W(a) R^{-1} F(0) .
$$




\section{Terminating barriers}

Often it is simpler and more convenient to work with defective processes. In order to retrieve identities for nondefective processes we can let the killing rates go to 0 . We follow this idea in the rest of the paper and provide comments concerning nondefective processes in Section 8 .

Let us present results for all the cases when there are no reflecting barriers.

Theorem 1. For a defective MAP, it holds that

$$
\begin{aligned}
u_{(-\infty, \infty)}(x) & =\mathrm{e}^{G x} H-W(-x)=H \mathrm{e}^{R x}-W(-x), \\
u_{(-\infty, b \mid}(x) & =\mathrm{e}^{G b} W(b-x)-W(-x), \\
u_{\mid-a, \infty)}(x) & =W(a) \mathrm{e}^{R(x+a)}-W(-x), \\
u_{|-a, b|}(x) & =W(a) W(a+b)^{-1} W(b-x)-W(-x),
\end{aligned}
$$

where $u_{I}(x)$ is a density of the measure $U_{I}(\mathrm{~d} x)$ on the corresponding interval; see (1).

In the case of a Lévy process this result is due to Bertoin [6], Bingham [7], and Suprun [27]. The methods employed in these papers are different from ours. In particular, Bertoin [6] relied on the Wiener-Hopf factorization. It should be noted that a Lévy process $H=1 / F^{\prime}(\Phi)$ yields

$$
u_{(-\infty, \infty)}(x)=\frac{1}{F^{\prime}(\Phi)} \mathrm{e}^{-\Phi x}-W(-x),
$$

which agrees with a representation appearing in [25, Theorem. 1].

In the case of an MMBM (with a nonzero Brownian component in each state) $\mathbb{P}\left[J\left(\tau_{\{x\}}\right)\right]$ is $\mathrm{e}^{G x}$ for $x \geq 0$ or $\mathrm{e}^{-\tilde{G} x}$ for $x<0$, where $\tilde{G}$ is the analogue of $G$ for $(-X, J)$ process. From (8) and (9), it follows that

$$
W(x)=\left(\mathrm{e}^{-G x}-\mathrm{e}^{\tilde{G} x}\right) H
$$

and that

$$
u_{(-\infty, \infty)}(x)=\left(\mathrm{e}^{G x} 1_{\{x \geq 0\}}+\mathrm{e}^{-\tilde{G} x} 1_{\{x<0\}}\right) H .
$$

The rest of the formulae can be rewritten in terms of $G, \tilde{G}$, and $H$.

Proof of Theorem 1. When computing the densities $u_{I}(x)$, we do not consider the case $x=0$, since $u_{I}(0)$ can be arbitrary. The potential measure of the free process $(X, J)$ is given by

$$
U_{i j}(A)=\mathbb{E}_{i} \int_{0}^{\infty} 1_{\{X(t) \in A, J(t)=j\}} \mathrm{d} t=\mathbb{E}_{i} \int_{A} L(x, j, \infty) \mathrm{d} x
$$

according to (4), and, hence, it has a density $u_{i j}(x)=\mathbb{E}_{i} L(x, j, \infty)$. By the additive property of $L$ and (10), we write

$$
u(x)=\mathbb{P}\left[J\left(\tau_{\{x\}}\right)\right] H=\mathrm{e}^{G x} H-W(-x) .
$$

Together with (6) this proves (12).

Similarly, we find that for $x<b$,

$$
\begin{aligned}
u_{(-\infty, b \mid}(x) & =\left(\mathbb{E}_{i} L\left(x, j, \tau_{b}^{+}\right)\right) \\
& =\mathbb{P}\left[J\left(\tau_{\{x\}}\right)\right] H-\mathbb{P}\left[J\left(\tau_{b}^{+}\right)\right] \mathbb{P}\left[J\left(\tau_{\{x-b\}}\right)\right] H \\
& =\mathrm{e}^{G x} H-W(-x)-\mathrm{e}^{G b}\left(\mathrm{e}^{G(x-b)} H-W(b-x)\right) \\
& =\mathrm{e}^{G b} W(b-x)-W(-x) .
\end{aligned}
$$


For $x>-a$, we have

$$
\begin{aligned}
u_{\mid-a, \infty)}(x) & =\left(\mathbb{E}_{i} L\left(x, j, \tau_{-a}^{-}\right)\right) \\
& =\mathbb{P}\left[J\left(\tau_{\{x\}}\right)\right] H-\mathbb{P}\left[J\left(\tau_{\{-a\}}\right)\right] \mathbb{P}\left[J\left(\tau_{a+x}^{+}\right)\right] H \\
& =\mathrm{e}^{G x} H-W(-x)-\left(\mathrm{e}^{-G a}-W(a) H^{-1}\right) \mathrm{e}^{G(x+a)} H \\
& =W(a) \mathrm{e}^{R(x+a)}-W(-x),
\end{aligned}
$$

where we used (6) and the fact that if a process has to pass over $-a$ and then return to $x>-a$ then it has to hit $-a$.

Finally, for $-a<x<b$, we write

$$
\begin{aligned}
u_{|-a, b|}(x)= & \left(\mathbb{E}_{i} L\left(x, j, \tau_{-a}^{-} \wedge \tau_{b}^{+}\right)\right) \\
= & \mathbb{P}\left[J\left(\tau_{\{x\}}\right)\right] H-\mathbb{P}\left[\tau_{b}^{+}<\tau_{\{-a\}}, J\left(\tau_{b}^{+}\right)\right] \mathbb{P}\left[J\left(\tau_{\{x-b\}}\right)\right] H \\
& -\mathbb{P}\left[\tau_{\{-a\}}<\tau_{b}^{+}, J\left(\tau_{\{-a\}}\right)\right] \mathbb{P}\left[J\left(\tau_{x+a}^{+}\right)\right] H .
\end{aligned}
$$

Observe that

$$
\mathbb{P}\left[\tau_{\{-a\}}<\tau_{b}^{+}, J\left(\tau_{\{-a\}}\right)\right]=\mathbb{P}\left[J\left(\tau_{\{-a\}}\right)\right]-\mathbb{P}\left[\tau_{b}^{+}<\tau_{\{-a\}}, J\left(\tau_{b}^{+}\right)\right] \mathbb{P}\left[J\left(\tau_{\{-a-b\}}\right)\right],
$$

and use (7) and (10) to obtain, after a number of cancellations,

$$
u_{|-a, b|}(\mathrm{d} x)=W(a) W(a+b)^{-1} W(b-x)-W(-x) .
$$

It is noted that (15) can be used to obtain all the other results in Theorem 1 by taking limits as $a, b \rightarrow \infty$. Computation of the limiting expressions is often not easy. We approach this by using Theorem 1 to state some useful limits.

Corollary 1. For a defective process and $y \geq 0$ the following limits hold as $x \rightarrow \infty$ :

$$
\begin{aligned}
\mathrm{e}^{G x} W(x) & \rightarrow H, \quad W(x) \mathrm{e}^{R x} \rightarrow H, \\
W(x) W(x+y)^{-1} \rightarrow \mathrm{e}^{G y}, & W(x+y)^{-1} W(x) \rightarrow \mathrm{e}^{R y} .
\end{aligned}
$$

Proof. The proof of Theorem 1 immediately implies that

$$
\lim _{a \rightarrow \infty} u_{\mid-a, \infty)}(x)=u_{(-\infty, \infty)}(x) \quad \text { for } x \neq 0 .
$$

Using (14) and (12), we see that $W(a) \mathrm{e}^{R a} \rightarrow H$ as $a \rightarrow \infty$. The other limits are obtained in a similar way.

\section{Time reversal}

Consider a nondefective MAP $(X, J)$, and let $\pi$ be the stationary distribution of $J$. Assuming that $J(0)$ has the distribution $\pi$, we define, for an arbitrary $t>0$, a time-reversed process by

$$
\hat{J}(s)=J((t-s)-), \quad \hat{X}(s)=X(t)-X((t-s)-),
$$

where $s \in[0, t)$. It is known that $(\hat{J}(s), \hat{X}(s))_{s \in[0, t)}$ is again a spectrally negative MAP (no downward subordinators among its components). This time-reversed MAP is characterized by

$$
\hat{F}(\alpha)=\Delta_{\pi}^{-1} F(\alpha)^{T} \Delta_{\pi},
$$


and it can be continued to $s \in[0, \infty)$. Now suppose that we kill both the original and the time-reversed processes using the same vector for the killing rates, $\boldsymbol{q}$. Then

$$
\hat{F}^{q}(\alpha)=\hat{F}(\alpha)-\Delta_{\boldsymbol{q}}=\Delta_{\pi}^{-1}\left(F(\alpha)-\Delta_{\boldsymbol{q}}\right)^{T} \Delta_{\pi}=\Delta_{\pi}^{-1} F^{q}(\alpha)^{T} \Delta_{\pi},
$$

i.e. the same identity is true, where $\pi$ corresponds to the nondefective transition rate matrix $F^{q}(0)+\Delta_{q}$. In the following we drop the superscript $\boldsymbol{q}$, where it does not lead to confusion, and use $\hat{\mathbb{P}}$ to denote the law of the (possibly killed) time-reversed process $(\hat{X}(s), \hat{J}(s))$. Importantly, time-reversed quantities can be expressed through their original analogues.

Proposition 1. The following identities hold

$$
\begin{aligned}
\hat{R} & =\Delta_{\pi}^{-1} G^{T} \Delta_{\pi}, & \hat{G} & =\Delta_{\pi}^{-1} R^{T} \Delta_{\pi}, \\
\hat{H} & =\Delta_{\pi}^{-1} H^{T} \Delta_{\pi}, & \hat{W}(x) & =\Delta_{\pi}^{-1} W(x)^{T} \Delta_{\pi} .
\end{aligned}
$$

Proof. The identities in the first line were obtained in [18]. In this paper we provide an alternative derivation. First, we compute using (17), i.e.

$$
\int_{0}^{\infty} \mathrm{e}^{-\alpha x} \Delta_{\pi}^{-1} W(x)^{T} \Delta_{\pi} \mathrm{d} x=\Delta_{\pi}^{-1}\left[F(\alpha)^{-1}\right]^{T} \Delta_{\pi}=\hat{F}(\alpha)^{-1},
$$

which establishes the result for $\hat{W}(x)$, because its transform identifies the continuous $\hat{W}(x)$ uniquely.

Next, we write

$$
\hat{W}(x) \hat{W}(x+y)^{-1}=\Delta_{\pi}^{-1}\left(W(x+y)^{-1} W(x)\right)^{T} \Delta_{\pi}
$$

and note that according to Corollary 1 the left-hand side converges to $\mathrm{e}^{\hat{G} y}$ and the right-hand side converges to

$$
\Delta_{\pi}^{-1}\left(\mathrm{e}^{R y}\right)^{T} \Delta_{\pi}=\mathrm{e}^{\Delta_{\pi}^{-1} R^{T} \Delta_{\pi} y} \quad \text { as } x \rightarrow \infty,
$$

which therefore should be equal for all $y \geq 0$. So $\hat{G}=\Delta_{\pi}^{-1} R^{T} \Delta_{\pi}$, which can be seen by e.g. integrating over $y \in(0, \infty)$. Swapping the roles of the time-reversed and the original processes we obtain $G=\Delta_{\pi}^{-1} \hat{R}^{T} \Delta_{\pi}$.

Finally, we consider

$$
\hat{W}(x) \mathrm{e}^{\hat{R} x}=\Delta_{\pi}^{-1} W(x)^{T} \mathrm{e}^{G^{T} x} \Delta_{\pi}=\Delta_{\pi}^{-1}\left(\mathrm{e}^{G x} W(x)\right)^{T} \Delta_{\pi},
$$

which, by letting $x \rightarrow \infty$ and using Corollary 1 , yields $\hat{H}=\Delta_{\pi}^{-1} H^{T} \Delta_{\pi}$. These results are obtained for defective processes, but can be easily extended to nondefective processes by letting the killing rates approach 0 .

Let us complement Corollary 1 with another useful limiting result, which also demonstrates how time-reversed quantities can be used to change the order of matrix multiplication.

Lemma 1. For a defective process the following limits hold as $x \rightarrow \infty$ :

$$
Z(x) F(0)^{-1} W(x)^{-1} \rightarrow G^{-1}, \quad W(x)^{-1} Z(x) F(0)^{-1} \rightarrow R^{-1} .
$$




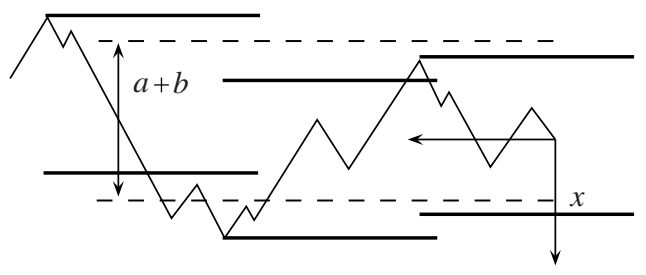

FIGURE 1: A sample path of $X$ with shifting barriers, and its time reversal according to the new axes.

Proof. Note that $W(x)^{-1}$ stays bounded as $x \rightarrow \infty$, because, for $x>1$, the matrix $W(1) W(x)^{-1}$ is a probability matrix. Now the second limit follows readily from (11). Using the relation $Z(x) F(0)^{-1}=F(0)^{-1}-\int_{0}^{x} W(y) \mathrm{d} y$, we write

$$
\Delta_{\pi}^{-1}\left(\hat{Z}(x) \hat{F}(0)^{-1}\right)^{T} \Delta_{\pi}=Z(x) F(0)^{-1}
$$

and, hence,

$$
Z(x) F(0)^{-1} W(x)^{-1}=\Delta_{\pi}^{-1}\left(\hat{W}(x)^{-1} \hat{Z}(x) \hat{F}(0)^{-1}\right)^{T} \Delta_{\pi} \rightarrow \Delta_{\pi}^{-1} \hat{R}^{-1} \Delta_{\pi}=G^{-1},
$$

which concludes the proof.

Let us present an application of time-reversal, which lays the basis for deriving a formula for the potential density in the case of two reflecting barriers.

Lemma 2. For a possibly defective $(X(s), J(s))$, any $t>0$, and $x \in[0, a+b]$, it holds that

$$
\begin{aligned}
\mathbb{P}_{a}\left[X_{[0, a+b]}(t) \geq x ; J(t)\right]= & \Delta_{\pi}^{-1}(\hat{\mathbb{P}}[\tau \leq t, X(\tau) \geq x ; J(t)] \\
& +\hat{\mathbb{P}}[\tau>t, X(t)+a \geq x ; J(t)])^{T} \Delta_{\pi} .
\end{aligned}
$$

where $\tau=\inf \{s \geq 0: X(s) \notin[x-a-b, x)\}$.

This type of identity was first noted in [23] in the case of a random walk with two reflecting barriers. A short derivation of its continuous-time analogue is given in [2, Proposition XIV.3.7]; see also [4] and [15] for the case of a Markov additive input. The above results concern stationary distribution. Here we allow for a finite time $t$ and a defective process.

Proof of Lemma 2. First assume that $(X(s), J(s))$ is nondefective. Similarly to the proof of [2, Proposition XIV.3.7], analysis of the sample paths of the original process (and, hence, of the corresponding time-reversed process) shows that

$$
\left\{X_{[0, a+b]}(t) \geq x, X(0)=a\right\}=\{\hat{\tau} \leq t, \hat{X}(\hat{\tau}) \geq x\} \cup\{\hat{\tau}>t, \hat{X}(t)+a \geq x\},
$$

where $\hat{X}(0)=0$. It is easy to understand this relation by considering a free process and shifting the reflecting boundaries as time evolves; see Figure 1. Then exit of $\hat{X}(s)$ from $[x-a-b, x)$ through $x$ means that the original process will not be able to shift the boundaries high enough immediately before time $t$ to make $X_{[0, a+b]}(t)<x$. In the same way exit over $x-a-b$ implies the converse. In the case of no exit from this interval, $X_{[0, a+b]}(t) \geq x$ if and only if $X(t) \geq x$, because the barriers cannot be shifted far enough. Conclude by observing that $X(t)=\hat{X}(t)+a$. 
Conditioning on the states of $J$ at times 0 and $t$, we arrive at

$$
\begin{aligned}
\mathbb{P}_{a, i}\left(X_{[0, a+b]}(t) \geq x \mid J(t)=j\right)= & \hat{\mathbb{P}}_{j}(\tau \leq t, X(\tau) \geq x \mid J(t)=i) \\
& +\hat{\mathbb{P}}_{j}(\tau>t, X(t)+a \geq x \mid J(t)=i),
\end{aligned}
$$

which immediately yields the result for a nondefective process.

Let us kill the original nondefective process using the killing rates $\boldsymbol{q}$. Given the whole evolution of the nondefective process on $[0, t]$ (and, hence, its time-reversed counterpart), the probability of no killing is

$$
\exp \left(-\sum_{i} q_{i} \int_{0}^{t} 1_{\{J(s)=i\}} \mathrm{d} s\right)
$$

Note that this expression remains the same if we substitute $\hat{J}$ instead of $J$. Finally, intersect both sides of (19) with the event of no killing in $[0, t]$, and condition on the evolution of the nondefective process to show that the result is also true in the presence of killing.

Remark 5. Lemma 2 simplifies, in the case of one-sided reflection $(b=\infty)$, to

$$
\mathbb{P}_{a}\left[X_{[0, \infty)}(t) \geq x ; J(t)\right]=\Delta_{\pi}^{-1}\left(\hat{\mathbb{P}}\left[\tau_{x}^{+} \leq t ; J(t)\right]+\hat{\mathbb{P}}\left[\tau_{x}^{+}>t, X(t)+a \geq x ; J(t)\right]\right)^{T} \Delta_{\pi} .
$$

If $a=0$ then the second term in brackets disappears and we have a well-known identity

$$
\mathbb{P}_{0}\left[X_{[0, \infty)}(t) \geq x ; J(t)\right]=\Delta_{\pi}^{-1} \hat{\mathbb{P}}[\bar{X}(t) \geq x ; J(t)]^{T} \Delta_{\pi},
$$

which can be explained by a very simple time-reversal argument.

\section{Reflecting barriers}

Theorem 2. For a defective MAP, it holds that the potential measure $U_{[-a, b]}(\mathrm{d} x)$ has a density

$$
u_{[-a, b]}(x)=-Z(a) F(0)^{-1} W(a+b)^{-1} W^{\prime}(b-x)-W(-x) \quad \text { on }(-a, b),
$$

and

$$
U_{[-a, b]}\{-a\}=\mathbb{O}, \quad U_{[-a, b]}\{b\}=-Z(a) F(0)^{-1} W(a+b)^{-1} W(0) .
$$

Proof. Take an independent RV $T \sim \operatorname{Exp}(q), q>0$. According to (3) and Lemma 2, we write, for $x \in[0, a+b]$,

$$
\begin{aligned}
U_{[-a, b]}^{q}[x-a, b]= & \frac{1}{q} \mathbb{P}_{a}\left[X_{[0, a+b]}(T) \geq x ; J(T)\right] \\
= & \frac{1}{q} \Delta_{\pi}^{-1}(\hat{\mathbb{P}}[\tau \leq T, X(\tau) \geq x ; J(T)] \\
& +\hat{\mathbb{P}}[\tau>T, X(T)+a \geq x ; J(T)])^{T} \Delta_{\pi} .
\end{aligned}
$$

Furthermore, using the fact that $\tau=\tau_{x}^{+} \wedge \tau_{x-a-b}^{-}$, we write

$$
\begin{aligned}
\mathbb{P}[\tau \leq T, X(\tau) \geq x ; J(T)] & =\mathbb{P}^{q}\left[\tau_{x}^{+}<\tau_{x-a-b}^{-} ; J\left(\tau_{x}^{+}\right)\right] \mathbb{P}[J(T)] \\
& =W^{q}(a+b-x) W^{q}(a+b)^{-1} q(q \mathbb{I}-Q)^{-1},
\end{aligned}
$$


where $W^{q}$ corresponds to a process with additional killing, of rate $q$, in each state. Moreover,

$$
\begin{aligned}
\mathbb{P}[\tau>T, X(T) \geq x-a ; J(T)]= & q \int_{x-a}^{x} u_{|x-a-b, x|}^{q}(y) \mathrm{d} y \\
= & q \int_{x-a}^{x} W^{q}(a+b-x) W^{q}(a+b)^{-1} \\
& \times W^{q}(x-y)-W^{q}(-y) \mathrm{d} y
\end{aligned}
$$

where in the second equality we use (15). Taking time-reversed quantities and using (18), we arrive at

$$
\begin{aligned}
U_{[-a, b]}^{q}[x-a, b]= & (q \mathbb{I}-Q)^{-1} W^{q}(a+b)^{-1} W^{q}(a+b-x) \\
& +\int_{x-a}^{x} W^{q}(x-y) W^{q}(a+b)^{-1} W^{q}(a+b-x)-W^{q}(-y) \mathrm{d} y \\
= & -Z^{q}(a) F^{q}(0)^{-1} W^{q}(a+b)^{-1} W^{q}(a+b-x) \\
& -\int_{(x-a)^{-}}^{0} W^{q}(-y) \mathrm{d} y .
\end{aligned}
$$

Finally, taking $q \downarrow 0$ we obtain

$$
U_{[-a, b]}[x, b]=-Z(a) F(0)^{-1} W(a+b)^{-1} W(b-x)-\int_{x^{-}}^{0} W(-y) \mathrm{d} y,
$$

which has a density on $(-a, b)$ and point masses as given in the statement of the theorem.

Remark 6. We may wonder about the asymmetry of point masses at $-a$ and at $b$. If we consider an MMBM (with nonzero Brownian components) then $W(0)=\mathbb{O}$ (according to Remark 4) implying that $U_{[-a, b]}\{b\}=\mathbb{O}$. In general, the $j$ th row of $U_{[-a, b]}\{b\}$ is a 0 vector if 0 is regular for itself under $\mathbb{P}_{j}$.

Corollary 2. For a defective MAP, it holds that

$$
\begin{aligned}
u_{[-a, b \mid}(x) & =Z(a) Z(a+b)^{-1} W(b-x)-W(-x), \\
u_{\mid-a, b]}(x) & =W(a) W_{+}^{\prime}(a+b)^{-1} W^{\prime}(b-x)-W(-x), \\
u_{[-a, \infty)}(x) & =Z(a) F(0)^{-1} R \mathrm{e}^{R(a+x)}-W(-x), \\
u_{(-\infty, b]}(x) & =-G^{-1} \mathrm{e}^{G b} W^{\prime}(b-x)-W(-x) .
\end{aligned}
$$

Additionally,

$$
\begin{aligned}
U_{[-a, b \mid}\{-a\} & =\mathbb{O}, \quad U_{\mid-a, b]}\{b\} & =W(a) W_{+}^{\prime}(a+b)^{-1} W(0), \\
U_{[-a, \infty)}\{-a\} & =\mathbb{O}, \quad U_{(-\infty, b]}\{b\} & =-G^{-1} \mathrm{e}^{G b} W(0) .
\end{aligned}
$$

In the case of a Lévy process this result is from [20], [24], with an alternative proof in [13].

Proof of Corollary 2. Proof of (21). Note that, for any Borel $A \subset[-a, b]$ we have

$$
U_{[-a, b]}(A)=U_{[-a, b \mid}(A)+\mathbb{P}\left[J\left(\underline{\sigma}_{-a, b}\right)\right] U_{[-a-b, 0]}(A-b),
$$


where $\underline{\sigma}_{-a, b}$ is the first passage time over level $b$ of a process reflected at $-a$. It is known that $\mathbb{P}\left[J\left(\underline{\sigma}_{-a, b}\right)\right]=Z(a) Z(a+b)^{-1}$; see [17, Theorem 2]. Hence, for $x \in(-a, b)$,

$$
\begin{aligned}
u_{[-a, b \mid}(x)= & u_{[-a, b]}(x)-Z(a) Z(a+b)^{-1} u_{[-a-b, 0]}(x-b) \\
= & -Z(a) F(0)^{-1} W(a+b)^{-1} W^{\prime}(b-x)-W(-x) \\
& +Z(a) Z(a+b)^{-1}\left(Z(a+b) F(0)^{-1} W(a+b)^{-1} W^{\prime}(b-x)+W(b-x)\right) \\
= & Z(a) Z(a+b)^{-1} W(b-x)-W(-x) .
\end{aligned}
$$

Moreover, $U_{[-a, b \mid}\{-a\}=\mathbb{O}$ is implied by $U_{[-a, b]}\{-a\}=\mathbb{O}$. There is no point mass at $b$, since the process exceeds $b$ immediately after hitting it.

Proof of (22). Similarly to the above, for $x \in(-a, b)$ we have

$$
u_{[-a, b]}(x)=u_{\mid-a, b]}(x)+\mathbb{P}\left[J\left(\bar{\sigma}_{-a, b}\right)\right] u_{[0, a+b]}(x+a),
$$

where $\bar{\sigma}_{-a, b}$ is the first passage below $-a$ of a process reflected at $b$. From [17, Theorem 6], we find that

$$
\mathbb{P}\left[J\left(\bar{\sigma}_{-a, b}\right)\right]=Z(a)+W(a) W_{+}^{\prime}(a+b)^{-1} W(a+b) F(0)
$$

and, hence,

$$
\begin{aligned}
u_{\mid-a, b]}(x)= & -Z(a) F(0)^{-1} W(a+b)^{-1} W^{\prime}(b-x)-W(-x) \\
& +\left(Z(a)+W(a) W_{+}^{\prime}(a+b)^{-1} W(a+b) F(0)\right) \\
& \times F(0)^{-1} W(a+b)^{-1} W^{\prime}(b-x) \\
= & W(a) W_{+}^{\prime}(a+b)^{-1} W^{\prime}(b-x)-W(-x) .
\end{aligned}
$$

Similarly, we find that $U_{\mid-a, b]}\{b\}=W(a) W_{+}^{\prime}(a+b)^{-1} W(0)$ and that there is no point mass at $-a$.

Finally, (23) is obtained from (21) by taking the limit as $b \rightarrow \infty$ and using Lemma 1 and Corollary 1. In particular, we use $Z(a+b)^{-1} W(a+b) \rightarrow F(0)^{-1} R$ and $W(a+b)^{-1} W(b-$ $x) \rightarrow \mathrm{e}^{R(a+x)}$. Moreover, (24) is obtained from (20) by letting $a \rightarrow \infty$, and the expressions for point masses follow similarly. Let us remark that (23) and (24) can be obtained directly using a time-reversal argument similar to that used in the proof of Lemma 2 (see also Remark 5).

We note that in the case of an MMBM the results of Theorem 2 and Corollary 2 can be rewritten using (16) (or its analogue if some of the Brownian components are nil; see, for example, [9]). The density $u_{[0, \infty)}(x)$ in the case of an MMBM was obtained in [10, Theorem 2] (there is a typographical error: $L$ should be at the end of the formula). The corresponding expression in [10] is rather lengthy, but it must simplify (assuming that $\left|E_{n}\right|=0$ in [10]) to $F(0)^{-1} R \mathrm{e}^{R x}$ according to (23). This simplification is difficult to achieve, because it may require nontrivial matrix algebra and various properties of the involved components; see, for example, [16, Section 7.7].

Finally, in the case of an MMBM various results can be obtained from their counterparts by considering an $\operatorname{MMBM}(\tilde{X}, \tilde{J})=(-X, J)$. In particular, $u_{(-\infty, 0]}(-x)$ should coincide with $\tilde{u}_{[0, \infty)}(x)$ for almost all $x \geq 0$. Indeed, using (16) and (24) we write

$$
u_{(-\infty, 0]}(-x)=-G^{-1}\left(-G \mathrm{e}^{-G x}-\tilde{G} \mathrm{e}^{\tilde{G} x}\right) H-\left(\mathrm{e}^{-G x}-\mathrm{e}^{\tilde{G} x}\right) H=\left(\tilde{G}^{-1}+G^{-1}\right) \tilde{G} \mathrm{e}^{\tilde{G} x} H,
$$

which should be compared to

$$
\tilde{u}_{[0, \infty)}(x)=\tilde{F}(0)^{-1} \tilde{R} \mathrm{e}^{\tilde{R} x}=\tilde{F}(0)^{-1} H^{-1} \tilde{G} \mathrm{e}^{\tilde{G} x} H
$$


according to (24), (6), and $\tilde{H}=H$, where the latter follows from the definition of $H$. It can be seen from [16, Section 7.7] that $\left(\tilde{G}^{-1}+G^{-1}\right) H=F(0)^{-1}=\tilde{F}(0)^{-1}$, which establishes the equality of the densities. Yet another check of our results is given in Section 9.

\section{Nondefective processes}

Let us discuss potential measures of a nondefective process. We may kill the process at rate $q>0$ and then let $q \downarrow 0$ to retrieve the original process. It is known that all the quantities $F(0), G, R, H, W(x), W^{\prime}(x)$, and $Z(x)$ are continuous in $q \geq 0$, and so the identities should still hold. The problem is that some limiting matrices may be infinite or singular, and that potential measures can be infinite as well. It is rather clear which cases are to be excluded; see also [2, Proposition XI.2.10].

Corollary 3. The results of Theorem 1 and Corollary 2 hold and, for a nondefective process apart from the following cases.

- $\mu<0$ : exclude (23).

- $\mu=0$ : exclude (12), (23), and (24).

- $\mu>0$ : exclude (24).

Moreover, when $\mu>0$, the term $F(0)^{-1} R$ in (23) should be understood in the limiting sense (as $q \downarrow 0)$.

Potential densities can also be used to obtain limiting distributions of reflected processes. According to (3) the limiting distribution is given by

$$
\lim _{q \downarrow 0} q U_{I}^{q}(\mathrm{~d} x),
$$

and, hence, it can be proper only if the limiting potential measure is infinite. In a certain sense these results will complement Corollary 3.

Let us first establish a simple lemma. We recall that $\pi$ is the stationary distribution of $J(t), t \geq 0$, and we let $\pi_{G}$ denote the stationary distribution of $J\left(\tau_{x}^{+}\right), x \geq 0$ when $\mu \geq 0$.

Lemma 3. For a nondefective process every row of $-\lim _{q \downarrow 0} q F^{q}(0)^{-1}$ equals $\pi$. If, in addition, $\mu \geq 0$ then every row of $-\lim _{q \downarrow 0} q\left(G^{q}\right)^{-1}$ equals $\mu \pi_{G}$.

Proof. Recall that $-q F^{q}(0)^{-1}=\mathbb{P}\left[J\left(e_{q}\right)\right]$, which immediately completes the proof of the first part. For the second part, compute

$$
-q\left(G^{q}\right)^{-1}=q \int_{0}^{\infty} \mathrm{e}^{G^{q} x} \mathrm{~d} x=\left(\mathbb{E}_{i} q \int_{0}^{\infty} 1_{\left\{\tau_{x}^{+}<e_{q}, J\left(\tau_{x}^{+}\right)=j\right\}} \mathrm{d} x\right)
$$

and observe that

$$
\frac{\int_{0}^{\infty} 1_{\left\{\tau_{x}^{+}<e_{q}, J\left(\tau_{x}^{+}\right)=j\right\}} \mathrm{d} x}{\bar{X}\left(e_{q}\right)} \rightarrow\left(\pi_{G}\right)_{j} \quad \text { a.s. }
$$

Furthermore, it is known that $\bar{X}(t) / t \rightarrow \mu$ a.s. as $t \rightarrow \infty$, and we can also put $e_{q}=e_{1} / q$. Hence, the proof is complete if we can show that the limit as $q \downarrow 0$ can be taken under the expectation sign in (25). This follows by the extended dominated convergence theorem and the fact that $\lim _{q \downarrow 0} \mathbb{E}\left(q \bar{X}\left(e_{q}\right)\right)=\mu$; see, for example, the proof of Corollary 2.1 of [3] for the latter. 
We are ready to present results concerning limiting distributions.

Corollary 4. The following limiting distributions exist and do not depend on the initial values $X(0)$ and $J(0)$, and are given by

$$
\begin{gathered}
\mathbb{P}_{i}\left[X_{[0, b]}(\infty) \in \mathrm{d} x, J(\infty)\right]=\pi W(b)^{-1}\left(W^{\prime}(b-x) \mathrm{d} x+W(0) \delta_{b}(\mathrm{~d} x)\right), \\
\mathbb{P}_{i}\left[X_{[0, \infty)}(\infty) \in \mathrm{d} x, J(\infty)\right]=-\pi R \mathrm{e}^{R x} \mathrm{~d} x, \quad \mu<0, \\
\mathbb{P}_{i}\left[X_{(-\infty, 0]}(\infty) \in \mathrm{d} x, J(\infty)\right]=\mu \pi_{G}\left(W^{\prime}(-x) \mathrm{d} x+W(0) \delta_{0}(\mathrm{~d} x)\right), \quad \mu>0,
\end{gathered}
$$

where $\delta_{b}$ denotes the Dirac measure on $b$.

Proof. The fact that these limiting distributions exist and do not depend on the initial values can be established in various ways; for example, we can use the time-reversal argument from Lemma 2. From Theorem 2 and (3), we see that

$$
\mathbb{P}\left[X_{[0, b]}(\infty) \geq x, J(\infty)\right]=-\lim _{q \downarrow 0} q F^{q}(0)^{-1} W(b)^{-1} W(b-x),
$$

which according to Lemma 3 yields

$$
\mathbb{P}_{i}\left[X_{[0, b]}(\infty) \geq x, J(\infty)\right]=\pi W(b)^{-1} W(b-x),
$$

and then the first result follows. The second and third results are obtained in a similar way using Corollary 2. We remark that these results can be obtained directly from Lemma 2.

Let us comment on the results of Corollary 4. Firstly, the first result has the following alternative form:

$$
\mathbb{P}_{i}\left[X_{[-a, 0]}(\infty) \in \mathrm{d} x, J(\infty)\right]=\pi W(a)^{-1}\left(W^{\prime}(-x) \mathrm{d} x+W(0) \delta_{0}(\mathrm{~d} x)\right) .
$$

The second and third results are known in the literature but in a different form. In particular, for $\mu<0$, it is known that $\left(X_{[0, \infty)}(\infty) \mid J(\infty)=i\right)$ has a phase-type distribution with transition rate matrix $\hat{G}$ started in state $i$; see, for example, [16, Corollary 2.23]. In other words, the column vector of densities is given by

$$
\mathbb{P}\left(X_{[0, \infty)}(\infty) \in \mathrm{d} x \mid J(\infty)\right)=\mathrm{e}^{\hat{G} x}(-\hat{G} \mathbf{1}) \mathrm{d} x,
$$

which leads to our result using Proposition 1. Considering the third result we compute the transform for large enough $\alpha>0$ :

$$
\int_{-\infty}^{0+} \mathrm{e}^{\alpha x} \mu \pi_{G}\left(W^{\prime}(-x) \mathrm{d} x+W(0) \delta_{0}(\mathrm{~d} x)\right)=\mu \pi_{G} \alpha F(\alpha)^{-1} .
$$

Here we used integration by parts to show that

$$
W(0)+\int_{0}^{\infty} \mathrm{e}^{-\alpha x} W^{\prime}(x) \mathrm{d} x=\alpha \int_{0}^{\infty} \mathrm{e}^{-\alpha x} W(x) \mathrm{d} x=\alpha F(\alpha)^{-1} .
$$

This confirms [16, Proposition 4.19], building upon [3]. 


\section{Concluding remarks}

\subsection{Exit from an open interval}

We may choose another way of terminating a process at a barrier. So far we have assumed that termination at $b$ occurs at the first exit from $(-\infty, b]$, and at $-a$ at the first exit from $[-a, \infty)$. We may choose to use open intervals $(-\infty, b)$ and $(-a, \infty)$ instead. Since $X(t)$ cannot hit a level without immediately passing over it, the result of Theorem 1 is still true apart from the case when $a=0$, i.e. the process is started at the boundary ( $b=0$ does not cause problems). In this case the process is killed at time 0 leading to 0 measures. Also, $u_{[-a, b \mid}(x)$ is still given by (21). There is however a substantial difference in the expression for $u_{\mid-a, b]}(x)$, because there might be an excursion of $X(t)$ from its maximum of height $a+b$ with positive probability; see [17, Theorem 5]. The new expression is given by

$$
\lim _{c \uparrow a} u_{\mid-c, b]}(x)=W(a) W_{-}^{\prime}(a+b)^{-1} W^{\prime}(b-x)-W(-x),
$$

where $W_{-}^{\prime}$ denotes a left derivative. So the formula remains the same if the left and right derivatives of $W$ coincide at $a+b$.

\subsection{Known transforms}

Some results are available in the literature that concern transforms of a one-sided reflection of $X(t)$ at an exponential time. In particular, [16, Corollary 4.21] states that

$$
\begin{aligned}
& -\frac{1}{q} \mathbb{E}\left[\mathrm{e}^{\alpha X\left(e_{q}\right)-(\alpha+\beta) \bar{X}\left(e_{q}\right)} ; J\left(e_{q}\right)\right] F^{q}(\alpha)=\mathbb{I}+(\alpha+\beta)\left(G^{q}-\beta \mathbb{I}\right)^{-1}, \\
& -\frac{1}{q} F^{q}(\alpha) \mathbb{E}\left[\mathrm{e}^{-\beta X\left(e_{q}\right)+(\alpha+\beta) \underline{X}\left(e_{q}\right)} ; J\left(e_{q}\right)\right]=\mathbb{I}+(\alpha+\beta)\left(R^{q}-\beta \mathbb{I}\right)^{-1},
\end{aligned}
$$

for $\alpha, \beta \geq 0$; see also [12] and [22] for results of a similar type. In particular, putting $\beta=0$ in the first equation and $\alpha=0$ in the second yields

$$
\begin{gathered}
-\int_{-\infty}^{0+} \mathrm{e}^{\alpha x} U_{(-\infty, 0]}(\mathrm{d} x) F(\alpha)=\mathbb{I}+\alpha G^{-1}, \\
-F(0) \int_{0-}^{\infty} \mathrm{e}^{-\beta x} U_{[0, \infty)}(\mathrm{d} x)=\mathbb{I}+\beta(R-\beta \mathbb{I})^{-1},
\end{gathered}
$$

where the common killing rate $q>0$ is implicit.

Let us check (23) and (24) against these results. Firstly, for large enough $\alpha>0$ we compute, similarly to (26),

$$
\int_{-\infty}^{0} \mathrm{e}^{\alpha x}\left(-G^{-1} W^{\prime}(-x)-W(-x)\right) \mathrm{d} x-G^{-1} W(0)=-\left(\alpha G^{-1}+\mathbb{I}\right) F(\alpha)^{-1} .
$$

Secondly, for $\beta \geq 0$, we compute

$$
\int_{0}^{\infty} \mathrm{e}^{-\beta x} F(0)^{-1} R \mathrm{e}^{R x} \mathrm{~d} x=-F(0)^{-1} R(R-\beta \mathbb{I})^{-1}=-F(0)^{-1}\left(\mathbb{I}+\beta(R-\beta \mathbb{I})^{-1}\right),
$$

which confirms the above result. 


\section{Acknowledgements}

This work was supported by the Swiss National Science Foundation Project 200020-143889 and the EU-FP7 project Impact2C. I would also like to express my gratitude to Zbigniew Palmowski, Runhuan Feng, and the anonymous referee for various helpful comments and suggestions at different stages of this manuscript.

\section{References}

[1] Albrecher, H. And Ivanovs, J. (2013). A risk model with an observer in a Markov environment. Risks 1, $148-161$.

[2] Asmussen, S. (2003). Applied Probability and Queues (Appl. Math. (New York) 51), 2nd edn. Springer, New York.

[3] Asmussen, S. and Kella, O. (2000). A multi-dimensional martingale for Markov additive processes and its applications. Adv. Appl. Prob. 32, 376-393.

[4] Asmussen, S. And Pihlsgård, M. (2007). Loss rates for Lévy processes with two reflecting barriers. Math. Operat. Res. 32, 308-321.

[5] Bertoin, J. (1996). Lévy Processes (Camb. Tracts Math. 121). Cambridge University Press.

[6] Bertoin, J. (1997). Exponential decay and ergodicity of completely asymmetric Lévy processes in a finite interval. Ann. App. Prob. 7, 156-169.

[7] Bingham, N. H. (1975). Fluctuation theory in continuous time. Adv. Appl. Prob. 7, 705-766.

[8] Breuer, L. (2008). First passage times for Markov additive processes with positive jumps of phase type. J. Appl. Prob. 45, 779-799.

[9] Breuer, L. (2012). Occupation times for Markov-modulated Brownian motion. J. Appl. Prob. 49, $549-565$.

[10] Breuer, L. (2013). The resolvent and expected local times for Markov-modulated Brownian motion with phase-dependent termination rates. J. Appl. Prob. 50, 430-438.

[11] D'Auria, B., Ivanovs, J., Kella, O. And MandJes, M. (2010). First passage of a Markov additive process and generalized Jordan chains. J. Appl. Prob. 47, 1048-1057.

[12] Dieker, A. B. And Mandjes, M. (2011). Extremes of Markov-additive processes with one-sided jumps, with queueing applications. Method. Comput. Appl. Prob. 13, 221-267.

[13] Doney, R. A. (2005). Some excursion calculations for spectrally one-sided Lévy processes. In Séminaire de Probabilités XXXVIII (Lecture Notes Math. 1857), Springer, Berlin, pp. 5-15.

[14] Feng, R. And Shimizu, Y. (2014). Potential measures of spectrally negative Markov additive processes with applications to ruin theory. Insurance Math. Econom. 59, 11-26.

[15] Ivanovs, J. (2010). Markov-modulated Brownian motion with two reflecting barriers. J. Appl. Prob. 47, $1034-$ 1047.

[16] Ivanovs, J. (2011). One-sided Markov additive processes and related exit problems. Doctoral Thesis, University of Amsterdam.

[17] Ivanovs, J. And Palmowski, Z. (2012). Occupation densities in solving exit problems for Markov additive processes and their reflections. Stoch. Process. Appl. 122, 3342-3360.

[18] Ivanovs, J. AND TAYloR, P. (2014). One-sided Markov additive processes and their lattice analogues. In preparation.

[19] Kella, O. (2006). Reflecting thoughts. Statist. Prob. Lett. 76, 1808-1811.

[20] Koroljuk, V. S., Suprun, V. N. And Šurenkov, V. M. (1976). A potential-theoretic method in boundary problems for processes with independent increments and jumps of the same sign. Teor. Verojatnost. Primenen. 21, 253-259 (in Russian).

[21] KYPRIANOU, A. E. (2006). Introductory Lectures on Fluctuations of Lévy processes with Applications. Springer, Berlin.

[22] Kyprianou, A. E. And Palmowski, Z. (2008). Fluctuations of spectrally negative Markov additive processes. In Séminaire de Probabilités XLI (Lecture Notes Math. 1934), Springer, Berlin, pp. 121-135.

[23] Lindley, D. (1959). Discussion on Mr. Winsten's paper. J. R. Statist. Soc. B 21, 22-23.

[24] Pistorius, M. R. (2004). On exit and ergodicity of the spectrally one-sided Lévy process reflected at its infimum. J. Theoret. Prob. 17, 183-220.

[25] Pistorius, M. R. (2005). A potential-theoretical review of some exit problems of spectrally negative Lévy processes. In Séminaire de Probabilités XXXVIII (Lecture Notes Math. 1857), Springer, Berlin, pp. 30-41.

[26] Salah, Z.B. and Morales, M. (2012). Lévy systems and the time value of ruin for Markov additive processes. Europ. Actuar. J. 2, 289-317.

[27] Suprun, V. N. (1976). The ruin problem and the resolvent of a killed independent increment process. Ukrainian Math. J. 28, 39-45. 